Instituto Internacional de Investigación y Desarrollo Tecnológico Educativo INDTEC, C.A.

DOI: https://doi.org/10.29394/Scientific.issn.2542-2987.2021.6.22.10.189-209

OAI-PMH: http://www.indteca.com/ojs/index.php/Revista Scientific/oai

Artículo Original / Original Article

\title{
Experiencias educativas frente al cambio climático. Un estudio con profesores universitarios
}

Autoras: Ana Lucía Maldonado González Universidad Veracruzana, UV anmaldonado@uv.mx Xalapa, México https://orcid.org/0000-0002-7780-8206

Ma. de los Ángeles Silva Mar Universidad Veracruzana, UV asilva@uv.mx Xalapa, México https://orcid.org/0000-0002-2186-1374

Sandra Luz Mesa Ortiz Universidad Veracruzana, UV smesa@uv.mx Xalapa, México

https://orcid.org/0000-0003-2962-2623

\section{Resumen}

Se presentan resultados de una investigación realizada con el objetivo de analizar posibilidades de educación para el Cambio Climático (CC) en experiencias educativas (EE) de nivel superior. El estudio se realiza a partir de una aproximación a la representación social del CC en profesores de la Universidad Veracruzana (UV), región Poza Rica, Tuxpan, México. Se aplicó un cuestionario a muestras representativas y se realizaron entrevistas grupales a profesores de las distintas áreas académicas. Los principales resultados se presentan aquí de manera descriptiva e interpretativa. Los participantes reconocen la gravedad del CC y los riesgos asociados a este fenómeno, particularmente en su región. También identifican posibilidades de acción desde sus EE, algunas de ellas ya implementadas tanto en espacios formales al interior del aula, pero también en espacios no formales, con actores comunitarios y públicos que participan de alguna forma en experiencias y prácticas académicas. Estas acciones contribuyen a la capacidad de adaptación y reducción de vulnerabilidades física, social y motivacional, con ello al fortalecimiento de la resiliencia social. Se reconoce así el compromiso social y ambiental en la transmisión del conocimiento y actitudes orientadas hacia la mitigación y adaptación al CC.

Palabras clave: educación ambiental; profesores; educación superior; vulnerabilidad; resiliencia social.

Código de clasificación internacional: 5802.07 - Formación profesional.

Cómo citar este artículo:

Maldonado, A., Silva, M., \& Mesa, S. (2021). Experiencias educativas frente al cambio climático. Un estudio con profesores universitarios. Revista Scientific, 6(22), 189-209, e-ISSN: 2542-2987. Recuperado de: https://doi.org/10.29394/Scientific.issn.2542-2987.2021.6.22.10.189-209

Fecha de Recepción: 21-01-2021
Fecha de Aceptación: 12-06-2021
Fecha de Publicación: 05-11-2021 
Instituto Internacional de Investigación y Desarrollo Tecnológico Educativo INDTEC, C.A.

DOI: https://doi.org/10.29394/Scientific.issn.2542-2987.2021.6.22.10.189-209

OAI-PMH: http://www.indteca.com/ojs/index.php/Revista Scientific/oai

Artículo Original / Original Article

\title{
Educational experiences in the face of climate change. A study with university professors
}

\begin{abstract}
This paper present results of a research about the possibilities of education for Climate Change (CC) in educational experiences (EE) of higher level. The study is carried out from an approach to the social representation of $\mathrm{CC}$ in professors from the Veracruzana University (UV), campus Poza Rica Tuxpan, Mexico. A questionnaire was applied to representative samples. Also group interviews were conducted with professors from the different academic areas. The main results are presented here descriptively and interpretively. Participants recognize the severity of $\mathrm{CC}$ and the risks associated to this phenomenon, particularly in their region. They also identify possibilities of action from theirs EE. Some of them already implemented, both in formal spaces, inside the classroom, but also in non-formal spaces where participate community and public actors in these academic experiences and practices. These actions contribute to the ability to adapt and reduce physical, social and motivational vulnerabilities, thereby strengthening social resilience. Thus, the social and environmental commitment is recognized, in the transmission of knowledge and attitudes oriented towards mitigation and adaptation to CC.
\end{abstract}

Keywords: environmental education; professors; higher education; vulnerability; social resilience.

International classification code: 5802.07 - Vocational education and training.

\footnotetext{
How to cite this article:

Maldonado, A., Silva, M., \& Mesa, S. (2021). Educational experiences against climate change. A study with university professors. Revista Scientific, 6(22), 189-209, e-ISSN: 2542-2987. Recovered from: https://doi.org/10.29394/Scientific.issn.2542-2987.2021.6.22.10.189-209
}

Date Received: 21-01-2021
Date Acceptance: 12-06-2021
Date Publication: 05-11-2021 


\section{Introducción}

Desde la educación es posible avanzar hacia una acción social y política comprometida y orientada a medidas de adaptación y mitigación al cambio climático (CC). Lo anterior en concordancia con la propuesta del Grupo Intergubernamental de Expertos sobre el Cambio Climático (IPCC, 2018): relacionada con promover y reforzar capacidades para la acción climática en autoridades, comunidades locales, poblaciones indígenas, actores privados, sociedad civil en general. De ahí la relevancia de la investigación que origina este artículo, al destacar la influencia de la educación y de los profesores universitarios, en este fortalecimiento de capacidades. El mismo IPCC reconoce el incremento en frecuencia y magnitud de fenómenos extremos como sequías, olas de calor, inundaciones, ciclones, incendios forestales, que se agravan, amenazan y afectan ecosistemas y poblaciones humanas mayormente expuestas y vulnerables a la crisis climática actual.

Si bien la alfabetización científica para González y Meira (2009): no garantiza cambios hacia comportamientos amigables con el ambiente, es importante reforzar el conocimiento sobre el cambio climático, pero dentro de una estrategia pedagógica considerando más bien una alfabetización ambiental ética, crítica dentro de la noción de ciudadanía y que sea ésta la que se impregne en la cultura común. En la medida en que un problema es reconocido por un mayor número de personas y se movilizan en consecuencia, el problema se socializa y con ello también las posibles soluciones, donde se involucran colectivamente distintos actores sociales. De ahí el interés por analizar y documentar lo que desde la educación superior se integra o se podría integrar sobre este fenómeno, en el aula como espacio formal, pero también en vinculación con otros actores sociales y desde el contexto comunitario, cuando las prácticas académicas así lo permiten.

A continuación, abordaremos en breve los fundamentos teóricoconceptuales que dan sustento a la investigación aquí presentada: el enfoque 
Instituto Internacional de Investigación y Desarrollo Tecnológico Educativo INDTEC, C.A.

DOI: https://doi.org/10.29394/Scientific.issn.2542-2987.2021.6.22.10.189-209

OAI-PMH: http://www.indteca.com/ojs/index.php/Revista Scientific/oai

Artículo Original / Original Article

procesual de las Representaciones Sociales (RS) y los conceptos de vulnerabilidad, riesgo y resiliencia social, mismos que por estar comprendidos en el contexto de estudio, fueron considerados para sustentar las categorías analíticas formales.

Para Moscovici (1979a): una representación social es una preparación para la acción y agrega que "[...] no lo es solo en la medida en que guía el comportamiento, sino sobre todo en la medida en que remodela y reconstituye los elementos del medio en el que el comportamiento debe tener lugar" (pág. 32). Es así que la representación, como modalidad específica del conocimiento, tiene la función de comunicación y elaboración de comportamientos entre individuos.

Desde el enfoque procesual de las representaciones sociales, Moscovici (1979b): explica que la dimensión de información corresponde a los conocimientos sobre un objeto, al interior de un grupo social. Aplicado esto a nuestra investigación, corresponde al conocimiento que sobre el CC tienen los profesores universitarios participantes y cómo lo obtienen. En cuanto a la dimensión campo de representación, se refiere a la imagen del objeto de estudio y la jerarquía que le es asignada. Finalmente, la dimensión de actitud, es la orientación favorable o no con respecto al objeto de estudio, otorgada por el grupo de participantes.

Diversas investigaciones cuyo objeto de estudio comprende la dimensión social del cambio climático, han recurrido al enfoque teórico y metodológico de las RS. Se identifican investigaciones con jóvenes de bachillerato como las de Bello, Alatorre y González-Gaudiano (2016); con jóvenes universitarios tales como Calixto (2018); Calixto y Terrón (2018); con futuros profesores mencionando a Gallego-Torres y Castro-Montaña (2020).

Como hallazgos relevantes en estas investigaciones, destaca que la información recibida por estas poblaciones sobre el fenómeno, proviene principalmente de internet, redes sociales y televisión; dicha información no 
garantiza cambios en estilos de vida o acciones sociales y políticas por el clima. Además, se trata de información no siempre certera, que puede derivar más bien en conocimiento erróneo o desconocimiento sobre el cambio climático, sus causas y consecuencias. Todo esto influye en las emociones y posibles respuestas activas o pasivas de la población, frente a este fenómeno complejo.

Tratándose además de una investigación que involucra conceptos como riesgo, vulnerabilidad y resiliencia, referimos a Beck (2000): quien explica que el riesgo se constituye a partir de una percepción social de los habitantes en un contexto específico, dicha percepción contribuye en la definición de pensamientos y acciones, aunque el riesgo adquiere realidad por sus efectos. Esto nos conduce a la asociación entre la percepción de riesgo y la vulnerabilidad propia del contexto.

Por su parte, Adger (1999): asocia la vulnerabilidad a la exposición que presenta una población en sus distintos medios de vida. Diversas tipologías de vulnerabilidad han sido definidas por varios autores, específicamente para nuestro estudio nos adherimos a la tipología de Anderson y Woodrow (1989): quienes destacan tres tipos de vulnerabilidad: 1). física (medio ambiente, clima, suelo, ingresos, tecnologías físicas, infraestructuras, viviendas); 2). social (estructura social, política, toma de decisiones, actividades sociales, económicas, liderazgo, raza, etnicidad, lenguaje, marginación, religión); 3). motivacional (la forma en que la comunidad se auto-percibe y enfrenta efectivamente adversidades en su contexto físico y social).

Desde la vulnerabilidad social y motivacional, pero sobre todo desde la forma en que éstas son superadas por una población y se aprende de una adversidad precisamente para avanzar en la reducción de vulnerabilidades, es como se va también fortaleciendo la resiliencia social. Diversas investigaciones previas de Mendoza (2017); Hernández (2017); y Avilés (2021): han sido abordadas considerando riesgo, vulnerabilidad y resiliencia. 
En estas investigaciones ha sido posible identificar a los jóvenes como agentes de cambio con altas posibilidades de influir en sus comunidades.

También manifiesta Cajigal (2018): que son reconocidos como figuras significativas que pueden influir y propiciar acciones de adaptación y mitigación al CC, entre sus estudiantes y también en distintos actores sociales, para gestionar riesgos, reducir vulnerabilidades y aumentar la resiliencia social.

De ahí que el objetivo de la investigación que genera este artículo, fue analizar la dimensión social del cambio climático en profesores universitarios, desde una aproximación a la representación social que tienen sobre el fenómeno, su integración y abordaje en sus prácticas y experiencias educativas, dentro y fuera del aula. En el siguiente apartado se describe el contexto tanto geográfico como institucional, así como la metodología y métodos que facilitaron la construcción y análisis de información, en la presente investigación.

\section{Metodología (Materiales y métodos)}

Por su ubicación geográfica colindante con el Golfo de México, los municipios de Poza Rica y Tuxpan, localizados en el extremo norte del estado de Veracruz, México, se encuentran altamente expuestos a fenómenos hidrometeorológicos extremos. Su proximidad geográfica entre sí, les permite compartir ecosistemas y también afectaciones derivadas de tormentas tropicales y huracanes, tales como inundaciones, deslaves y desbordamientos.

Además, se encuentran expuestos a diversos riesgos emanados de la actividad humana, como lo es la contaminación de la industria petroquímica y termoeléctrica localizada en la región como principal actividad económica. En estos municipios se encuentra una de las cinco sedes de la Universidad Veracruzana (UV, 2019): en la cual se realizó la investigación aquí presentada. La planta académica en esta sede se conforma por 548 docentes, para una 
matrícula de 8 mil 267 alumnos.

Desde la Universidad Veracruzana (UV, 2020): la gestión del riesgo es promovida a partir del Sistema Universitario de Gestión Integral del Riesgo (SUGIR), creado en el año 2013 con el fin de "[...] establecer y desarrollar la capacidad institucional para responder y contribuir permanentemente en la construcción de una cultura del autocuidado y del cuidado colectivo, desde una filosofía de gestión integral del riesgo congruente con la sustentabilidad" (pág. 1). En este programa se alerta continuamente sobre posibles riesgos del momento y la forma de prevenirnos o reaccionar con las medidas de seguridad y de precaución necesarias.

En este contexto de vulnerabilidades y riesgos, pero también de espacios educativos que promueven la gestión del riesgo y la resiliencia, la dimensión social del CC fue analizada en un grupo de profesores, desde el enfoque procesual de las representaciones sociales, según Moscovici (1979c): considerando para ello sus tres dimensiones: información, campo de representación y actitudes. Los resultados presentados en este artículo enfatizan las dimensiones de información y actitudes, esta última específicamente comprendida en procesos educativos que llevan a cabo los profesores participantes.

El enfoque metodológico es descriptivo e interpretativo, partimos de una realidad exterior pero además de aquella interiorizada en conocimientos previos, la cual se ve influenciada por las experiencias educativas que imparten y, para este estudio, también por el intercambio de los profesores participantes en distintas etapas de la investigación. En este contexto, para Lotz-Sisitka, Fien y Ketlhoilwe (2013): se trata de una realidad construida socialmente con distintos significados.

Se utilizaron métodos mixtos: cuestionario y entrevistas grupales. Primero se aplicó un cuestionario a muestras representativas de profesores, mismo que fue validado mediante prueba piloto, alcanzando un valor Alfa de 
Cronbach de 0.84 . El cuestionario se integra de preguntas cerradas y abiertas que exploran las tres dimensiones de las representaciones sociales (RS) desde su enfoque procesual.

Se realizó un muestreo proporcional y la muestra estuvo conformada por un total de 109 profesores de las siguientes áreas académicas de esta región: Ciencias de la salud, Humanidades, Técnica, Económico y Administrativa. Además, acorde con Fontana y Frey (2015): se aplicaron entrevistas grupales semiestructuradas para construir datos a partir de la interrogación sistemática y simultánea de varios profesores, lo que permitió obtener detalladas descripciones y experiencias compartidas.

En dichas entrevistas participaron en total dieciocho profesores divididos en cuatro grupos de acuerdo con su área académica, con el fin de profundizar en aspectos relacionados con la dimensión de actitudes, en relación con la forma en que abordan o podrían abordar el fenómeno del CC en sus experiencias educativas. Las categorías analíticas predefinidas para la entrevista fueron, desde la dimensión de información: 1). Amenazas identificadas en su región y asociadas al CC; y 2). Vulnerabilidad. Desde la dimensión de actitudes: 1). Gestión del riesgo; y 2). Resiliencia social.

A partir de esto se buscó analizar procesos sociales asociados al CC y presentes en las actividades académicas. Estas entrevistas grupales fueron transcritas textualmente para proceder a su análisis de contenido. Para organizar los datos y permitir el análisis sistemático de la información derivada de ambos métodos, cuestionario y entrevistas grupales, se recurrió a los softwares IBM SPSS y Atlas.ti, respectivamente. A continuación, presentamos los principales hallazgos.

\section{Resultados (análisis e interpretación de los resultados)}

Presentamos en un primer apartado los resultados correspondientes a la dimensión información sobre $\mathrm{CC}$, donde los profesores reconocen 
amenazas y vulnerabilidades asociadas al fenómeno en su contexto; en un siguiente apartado, se aborda lo correspondiente a la dimensión actitudes y cómo son éstas orientadas hacia la gestión del riesgo y la resiliencia social, lo anterior desde sus experiencias educativas que describen los profesores en las entrevistas grupales, reconociendo en ellas prácticas de educación para el cambio climático.

\subsection{Dimensión información sobre CC. Reconocimiento de amenazas y vulnerabilidades en el contexto}

Las consecuencias del fenómeno ya se padecen en la región de Poza Rica, Tuxpan, así lo afirma 95\% de los participantes en la investigación; 81\% afirma que se trata de un problema prioritario para México y para Veracruz. Reconocer un problema es el primer paso para actuar en consecuencia y, tratándose de profesores universitarios, una de estas acciones desde la academia tiene que ver con la comunicación y la educación para el cambio climático, tanto en espacios formales, desde el aula con sus alumnos, como en espacios no formales en vinculación con otros actores sociales.

Destacan como fuentes de información y comunicación sobre cambio climático: internet y redes sociales $90 \%$. Además, para informarse sobre este fenómeno recurren a documentales $81 \%$, noticieros $79 \%$, textos científicos $71 \%$, programas de televisión $68 \%$ y prensa $54 \%$. Ciertamente varían los niveles de veracidad y confiabilidad de la información procedente de las distintas fuentes, por lo que resulta relevante para este segmento poblacional de profesores, distinguir la información científica y sustentada, de aquella que busca ser alarmista y no tiene alcances formativos hacia la adaptación y mitigación al CC. 
Tabla 1. Información y comunicación sobre cambio climático.

\begin{tabular}{|lc|ll|}
\hline \multicolumn{1}{|c|}{$\begin{array}{c}\text { Fuentes de información sobre } \\
\text { cambio climático }\end{array}$} & $\%$ & Con quiénes habla sobre asuntos & $\%$ \\
\hline Internet y redes sociales & 90 & Con pares y colegas de la Universidad & 82 \\
Documentales & 81 & Con alumnos & 81 \\
Noticieros & 79 & Con familiares & 80 \\
Textos científicos & 71 & Con amistades & 78 \\
Televisión & 68 & En eventos académicos & 60 \\
Prensa & 54 & & \\
\hline
\end{tabular}

Fuente: Elaboración propia a partir de resultados.

La tabla 1 incluye las fuentes de información que consultan los profesores sobre este fenómeno, así como con quienes interaccionan sobre asuntos relacionados con el cambio climático. La mayoría de los participantes $82 \%$ afirma tener conversaciones sobre CC con sus pares, colegas en la misma universidad, pero también con sus alumnos $81 \%$, con familiares $80 \%$, amistades $78 \%$ y en eventos académicos $60 \%$. Es decir, hablan sobre CC tanto de manera formal al interior de sus cursos, como en espacios y tiempos fuera del aula, de manera informal. Socializar el problema es visibilizarlo, invitar a la acción, propiciarla colaborativamente. De ahí la importancia de retomar en el aula y fuera de ella información y conocimientos confiables sobre el fenómeno, realizar un análisis crítico, sugerir propuestas para la adaptación y mitigación.

Como se presentó de manera contextual, por su situación geográfica la región de Poza Rica, Tuxpan, se ve afectada por fenómenos extremos, mismos que pueden ser asociados a causas y consecuencias del CC, de ahí que se trata de una región vulnerable y expuesta a diversas amenazas. La mayoría de los profesores identifican entre éstas, las siguientes: mala calidad del aire y del agua 94\%; mayor exposición a hidrometeoros como tormentas intensas, ciclones y huracanes $93 \%$; mayor exposición a enfermedades transmitidas por vectores $90 \%$; riesgo de inundaciones $88 \%$; alteraciones al litoral de su región $82 \%$; restricciones de agua con mayor frecuencia $84 \%$. 
Además, reconocen amenazas a su economía familiar al reflejar en sus bolsillos un mayor gasto de energía por uso de ventiladores y aire acondicionado $95 \%$, así como la necesidad de invertir en la vivienda para ahorrar energía $89 \%$. Lo anterior derivado del incremento en la temperatura y olas de calor extremo que se registran en la región y que van en aumento.

De ahí que la mayoría de los participantes reconoce que los veranos son más largos y calurosos $92 \%$, que la temporada de invierno ahora es más seca y calurosa $85 \%$, que los periodos de sequía son más prolongados $89 \%$; que se están perdiendo cultivos y animales de corral $87 \%$; que se están extinguiendo plantas y animales $84 \%$. Es así como las amenazas identificadas por la mayoría de los participantes en su contexto, muestran un escenario de vulnerabilidad física y social que amerita acciones inmediatas de parte de todos los actores sociales.

\subsection{Dimensión de actitudes. Hacia la gestión del riesgo y resiliencia social}

Al cuestionar a los participantes sobre el despliegue de acciones en respuesta ante el cambio climático por parte de distintos actores sociales, la mayoría adjudica más responsabilidad a los gobiernos de cada país $89 \%$, al sector industrial $87 \%$ y a los países industrializados $87 \%$. Sin embargo, también $69 \%$ reconoce a las universidades como un actor social desde donde se podrían desplegar acciones de respuesta orientadas a gestionar el riesgo, reducir vulnerabilidades y avanzar en el proceso de resiliencia social. De ahí la relevancia de analizar lo que están realizando precisamente desde la universidad, a partir de sus experiencias educativas.

Casi todos los participantes 95\%, consideran relevante abordar el fenómeno del cambio climático en las distintas formaciones profesionales. Se trata de un problema frente al que toda la población puede actuar y particularmente los profesores tienen un compromiso social, por su papel de 
figuras significativas, con influencia en sus alumnos y en su comunidad.

A partir del reconocimiento que ya tienen los profesores participantes sobre el cambio climático, sus implicaciones y relevancia por abordar el fenómeno, se cuestiona en la investigación sobre las actividades de mitigación y adaptación al CC que ya organizan o en las cuales se involucran, resultando lo siguiente: $64 \%$ ha implementado en su hogar alguna actividad de adaptación; $45 \%$ ha participado en actividades universitarias o extraescolares que privilegian la mitigación y adaptación al CC; $33 \%$ ha organizado alguna actividad universitaria en este sentido y $26 \%$ afirma haber implementado también entre sus vecinos actividades afines. Se reconoce así un compromiso en lo individual y familiar, pero falta todavía desplegar más acciones en su comunidad y en la universidad. En cuanto al abordaje del cambio climático en sus experiencias educativas (EE), $48 \%$ de los participantes reconoce que incluye este fenómeno en las EE que imparte.

Asimismo, sobre su disponibilidad para desarrollar actividades de mitigación y adaptación al cambio climático desde su práctica docente, más de $85 \%$ de los participantes manifiesta su interés por integrar el cambio climático en distintos espacios, tales como: 1). participar en programas de formación docente y diseño de actividades académicas sobre este fenómeno; 2). colaborar en distintos programas universitarios sobre CC; 3 ). realizar en cuerpos colegiados un diagnóstico participativo sobre la incorporación del cambio climático en diversas actividades académicas; y 4). su incorporación en las EE que ya imparte, así como problemas asociados a sus causas y consecuencias, particularmente el riesgo de inundación que afecta a esta región. A continuación, algunos ejemplos donde los profesores reconocen esta incorporación tanto dentro del aula como en vinculación con comunidades y actores sociales.

Desde la licenciatura en Psicología, los estudiantes realizan prácticas profesionales en vinculación con comunidades que han sufrido los embates de 
inundaciones. Mediante un proceso reflexivo de la experiencia vivida, así como a través de recorridos locales, es posible co-construir mapas de riesgos y documentar la memoria histórica comunitaria.

Lo anterior para generar un manual de difusión sobre prevención de riesgo de inundación. El proceso de reflexión y documentación sistemática de experiencias comunitarias antes, durante y después de una inundación, sin duda permite aprendizajes para todos los participantes, además de contribuir al autocuidado y autoestima comunitaria, factores relevantes para superar la vulnerabilidad social y motivacional, así como para reforzar la resiliencia social.

También las licenciaturas de Pedagogía y Trabajo Social privilegian que sus estudiantes realicen proyectos de intervención en escuelas y comunidades. Lo anterior una vez que cuentan con los conocimientos previos para abordar estas problemáticas frente a grupos. El objetivo es difundir medidas de prevención de riesgos de inundación desde un enfoque participativo, colaborativo, en el que todos aprenden de todos.

En el caso de la licenciatura en Biología, uno de los abordajes que hacen del cambio climático, es a partir de un análisis histórico de las temperaturas y su correlación con los cambios registrados en los ecosistemas locales. Además, para abordar el tema de inundaciones, solicitan que los estudiantes pregunten a sus padres y abuelos sus experiencias del pasado frente a este fenómeno. Dichas experiencias luego son compartidas y documentadas en el aula. Esto contribuye al aprendizaje reflexivo, al fortalecimiento de capacidades locales, a la disminución de vulnerabilidades y al proceso de resiliencia social. Se fomenta así el desarrollo de pensamiento crítico y creativo para actuar frente a situaciones de riesgo a las que están expuestos en su región.

Reducir vulnerabilidades ante inundaciones y olas de calor intenso son objetivos que buscan desde esta licenciatura en Arquitectura, esto contribuye a la promoción de medidas de mitigación y adaptación al CC. Lo anterior a 
partir de fortalecer desde sus experiencias educativas, habilidades y conocimientos de diseño y desarrollo de infraestructuras, análisis previo de tipos de suelos y materiales para la construcción de viviendas sustentables (con biodigestores, ecotecnias, separación de aguas, recursos de bajo consumo de agua). Esto permite en sus estudiantes una formación orientada a la arquitectura sustentable en todos los procesos.

La contaminación atmosférica en la región es analizada desde las licenciaturas de Ciencias Químicas, Ingeniería Química, Ingeniería Petrolera, Ingeniería Ambiental. Profesores y estudiantes en vinculación con agentes de gobierno participan en planes de acción climática municipal. De esta forma los estudiantes realizan análisis de inventarios de emisiones de gases de efecto invernadero, involucrándose en estudios de problemas reales, donde tienen la posibilidad de colaborar en procesos de toma de decisiones con agentes de nivel municipal.

Por su parte, profesores del Área Económica-Administrativa reconocen las posibilidades que tienen sus estudiantes de vincularse con empresarios, a partir de algunas experiencias educativas diseñadas con el fin de fomentar la implementación de protocolos que se adhieran a las regulaciones ambientales.

Son así identificadas prácticas reflexivas y colaborativas de profesores y estudiantes en vinculación con distintos actores sociales orientadas hacia una educación para el cambio climático, sugerido por diversos autores como Pruneau, Demers y Khattabi (2008); González y Meira (2020). Sin duda estas prácticas contribuyen a la gestión del riesgo, a reducir la vulnerabilidad física, social y motivacional en su contexto, así como a avanzar en el proceso de resiliencia social.

\section{Conclusiones}

Frente a un fenómeno complejo como el cambio climático, el reto educativo también lo es. Los profesores pueden ser considerados figuras 
significativas y los jóvenes agentes de cambio. Estamos frente a dos segmentos poblacionales relevantes en materia de educación para el CC. Los hallazgos permiten documentar la información que tienen los profesores participantes relacionada con este fenómeno, sus fuentes de información y de socialización, así como las principales amenazas asociadas que identifican en su región.

Se reconocen además actividades académicas realizadas en concordancia con lo que involucra una educación para el CC. Por mencionar algunas de las varias enlistadas previamente en el artículo, nos referimos a las EE donde involucran intervenciones comunitarias en las que profesores, estudiantes y comunidades actúan colaborativamente para que nuevas competencias relacionadas con el cambio climático sean comprendidas e integradas en acciones concretas, tales como elaboración de mapas de riesgo, planes de contingencia, documentación de cambios en ecosistemas naturales y humanos, prácticas de responsabilidad socioambiental. Todo esto contribuye para integrar al currículo materiales didácticos, desde una pedagogía que privilegia la alfabetización ambiental ética, crítica, política, la toma de decisiones y acciones respetuosas de nuestro entorno.

Sin duda se quedan fuera de este artículo muchas otras experiencias educativas donde integran el cambio climático los profesores de las distintas áreas académicas de la región Poza Rica, Tuxpan de la Universidad Veracruzana de México. Recuperarlas, documentarlas y reflexionar sobre ellas es también un proceso en continua co-construcción, del cual se derivan aprendizajes experienciales siempre favorables al fortalecimiento de capacidades para la acción climática.

\section{Referencias}

Adger, W. (1999). Social Vulnerability to Climate Change and Extremes in Coastal Vietnam. World Development, 27(2), 249-269, e-ISSN: 0305- 
Instituto Internacional de Investigación y Desarrollo Tecnológico Educativo INDTEC, C.A.

DOI: https://doi.org/10.29394/Scientific.issn.2542-2987.2021.6.22.10.189-209

OAl-PMH: http://www.indteca.com/ojs/index.php/Revista Scientific/oai

Artículo Original / Original Article

750X. Recovered from: https://doi.org/10.1016/S0305-750X(98)00136-

$\underline{3}$

Anderson, M., \& Woodrow, P. (1989). Rising from the ashes. Development

strategies in times of disaster. ISBN: 0-8133-7828-1. Colorado, United States: Westview Press.

Avilés, P. (2021). La construcción social del riesgo ambiental en estudiantes de bachillerato en Paso del Macho, Veracruz. Tesis. México: Universidad Veracruzana.

Beck, U. (2000). Retorno a la teoría de la sociedad del riesgo. Boletín de la Asociación de Geógrafos Españoles, (30), 9-20, e-ISSN: 2605-3322. Recuperado de:

https://bage.age-geografia.es/ojs/index.php/bage/article/view/383

Bello, L., Alatorre, G., \& González-Gaudiano, É. (2016). Representaciones Sociales sobre Cambio Climático. Un Acercamiento a sus Procesos de Construcción. Trayectorias, 18(43), 73-92 e-ISSN: 20071205. Recuperado de:

https://www.redalyc.org/articulo.oa?id=60746482004

Cajigal, E. (2018). Vulnerabilidad y resiliencia social ante inundaciones derivadas de ciclones tropicales en tres municipios de Veracruz. Un estudio con docentes de nivel bachillerato. Tesis. México: Universidad Veracruzana.

Calixto, R. (2018). El cambio climático en las representaciones sociales de los estudiantes universitarios. Redie: Revista Electrónica de Investigación Educativa, 20(1), 122-132, e-ISSN: 1607-4041. Recuperado de: https://doi.org/10.24320/redie.2018.20.1.1443

Calixto, R., \& Terrón, E. (2018). Las emociones en las representaciones sociales del cambio climático. Educar em Revista, 34(68), 217-233, e-ISSN: 1984-0411. Recuperado de: https://doi.org/10.1590/0104$\underline{4060.55684}$ 
Fontana, A., \& Frey, J. (2015). La entrevista. En Denzin, N., \& Lincoln, Y. (coords.). Manual de Investigación Cualitativa Volumen IV. Métodos de recolección y análisis de datos. (págs. 140-202). ISBN: 978-84-9784311-9. Barcelona, España: Editorial Gedisa.

Gallego-Torres, A., \& Castro-Montaña, J. (2020). Estudio de las representaciones sociales de los docentes sobre el cambio climático antropogénico. Revista Científica, 38(2), 229-242, e-ISSN: 2344-8350. Recuperado de: https://doi.org/10.14483/23448350.16190 González, E., \& Meira, P. (2020). Educación para el cambio climático. ¿Educar sobre el clima o para el cambio?. Perfiles Educativos, 42(168), 157-174, e-ISSN: 2448-6167. Recuperado de: https://doi.org/10.22201/iisue.24486167e.2020.168.59464

González, E., \& Meira, P. (2009). Educación, comunicación y cambio climático. Resistencias para la acción social responsable. Trayectorias, 11(29), 6-38, e-ISSN: 2007-1205. Recuperado de: http://www.redalyc.org/articulo.oa?id=60712749003

Hernández, D. (2017). Vulnerabilidad, riesgo y resiliencia ante fenómenos hidrometeorológicos en jóvenes de bachillerato de La Antigua, Veracruz. Tesis de Maestría en Investigación Educativa. México: Universidad Veracruzana. Recuperado de:

http://cdigital.uv.mx/handle/123456789/47401

IPCC (2018). Calentamiento global de $1,5^{\circ} \mathrm{C}$. Unidad de Soporte Técnico del Grupo de Trabajo I. Suiza: Grupo Intergubernamental de Expertos sobre el Cambio Climático.

Lotz-Sisitka, H., Fien, J., \& Ketlhoilwe, M. (2013). Traditions and New Niches: An Overview of Environmental Education Curriculum and Learning Research. In Stevenson, R., Brody, M., Dillon, J., \& Wals, A. (ed.). International handbook of research on environmental education. (pp. 194-205). ISBN: 978-0-415-89238-4. New York, United States: 
American Educational Research Association (AERA) by Routledge Publishers.

Mendoza, F. (2017). Estudio sobre programas de prevención de riesgo por inundaciones y su influencia en jóvenes de bachillerato de la localidad de José Cardel. Tesis de Maestría en Investigación Educativa. México: Universidad Veracruzana. Recuperado de:

http://cdigital.uv.mx/handle/123456789/47402

Moscovici, S. (1979a,b,c). El psicoanálisis, su imagen y su público. Buenos Aires, Argentina: Editorial Huemul.

Pruneau, D., Demers, M., \& Khattabi, A. (2008). Éduquer et communiquer en matière de changements climatiques: Défis et possibilités. VertigO: La revue électronique en sciences de l'environnement, 8(2), eISSN: 1492-8442. Recuperado de:

https://id.erudit.org/iderudit/019971ar UV (2020). Sistema Universitario de Gestión Integral del Riesgo (SUGIR). México: Universidad Veracruzana.

UV (2019). II Informe de Actividades 2018-2019: Pertenencia y Pertinencia. Programa de Trabajo Estratégico 2017-2021. México: Universidad Veracruzana. 


\section{Ana Lucía Maldonado González}

e-mail: anmaldonado@uv.mx

Nacida en Monterrey, Nuevo León, México, el 20 de

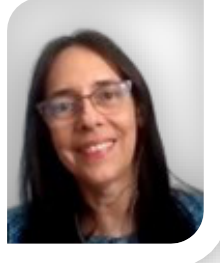
diciembre del año 1963. Académica del Instituto de Investigaciones en Educación de la Universidad Veracruzana (UV); Adscrita a la línea de investigación Educación Ambiental para la Sustentabilidad, desde la cual dirijo tesis de la Maestría y el Doctorado en Investigación Educativa, ambos programas de postgrado de excelencia del Consejo Nacional de Ciencia y Tecnología (CONACYT); Miembro del Sistema Nacional de Investigadores; Coordinadora del proyecto ganador del Premio a la Investigación Interdisciplinaria UV 2020-2021; Doctora en Trabajo Social por la Universidad Laval (ULaval), Quebec, Canadá; Temas de investigación: educación y comunicación ambiental, vulnerabilidad y resiliencia social, cambio climático, gobernanza ambiental, representaciones sociales. 
Ma. de los Ángeles Silva Mar

e-mail: asilva@uv.mx

Nacida Tampico, Tamaulipas, México, el 25 de septiembre del año 1961. Doctora en Gestión Ambiental para el Desarrollo por la Universidad Popular Autónoma de Veracruz (UPAV); Maestra en Educación, con especialidad en metodología de la enseñanza superior, Psicóloga educativa; Académica en la Facultad de Pedagogía de la Universidad Veracruzana (UV); Integrante del cuerpo académico INES; Línea de investigación: Innovación Educativa y Sustentabilidad; Docente de la Maestría en Gestión del Aprendizaje en el Programa Nacional de Posgrados de Calidad; Consejo Nacional de Ciencia y Tecnología (PNPC-CONACYT); Académica integrante de la Cátedra de la Organización de las Naciones Unidas para la Educación, la Ciencia y la Cultura (UNESCO) de Ciudadanía, Educación y Sustentabilidad Ambiental del Desarrollo en la Universidad Veracruzana. 


\section{Sandra Luz Mesa Ortiz \\ e-mail: smesa@uv.mx}

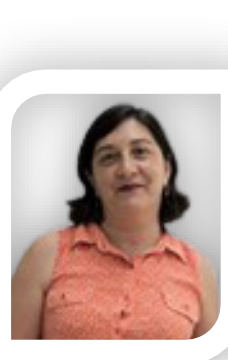

Nacida en Naolinco, Veracruz, México, el 14 noviembre del año 1964. Maestra en Estudios Regionales Medio Ambiente y Desarrollo (MERMAD) por la Universidad Iberoamericana Puebla (IBERO Puebla); Colaboradora y responsable en proyectos de investigación financiados por el Consejo Nacional de Ciencia y Tecnología (CONACYT); Ganadora del Premio a la Investigación Interdisciplinaria de la Universidad Veracruzana (UV); Coordinadora de la Maestría en Investigación Educativa (MIE); Académica del Instituto de Investigación en Educación (IIE), de la Universidad Veracruzana.

El contenido de este manuscrito se difunde bajo una Licencia de Creative Commons ReconocimientoNoComercial-Compartirlgual 4.0 Internacional 